\title{
High mobility organic transistor patterned by the shadow-mask with all structure on a plastic substrate
}

Joo-Won Lee $\cdot$ Byeong-Kwon Ju $\cdot$ Jin Jang $\cdot$

Young-Soo Yoon $\cdot$ Jai-Kyeong Kim

Published online: 22 March 2007

(C) Springer Science+Business Media, LLC 2007

Erratum to: J Mater Sci

DOI 10.1007/s10853-006-1046-z

The Publisher apologizes for a misprint that appeared in Journal of Materials Science, Volume 42, Number 3, pages 1026-1030 The corresponding authors for article "High mobility organic transistor patterned by the shadowmask with all structure on a plastic substrate" should be listed as Young-Soo Yoon and Jai-Kyeong Kim, not JooWon Lee.
The online version of the original article can be found at http:// dx.doi.org/10.1007/s10853-006-1046-z

J.-W. Lee · J.-K. Kim $(\bowtie)$

Opto-Electric Materials Research Center, Korea Institute of Science and Technology, Seoul 136-791, Korea

e-mail: jack@kist.re.kr

J.-W. Lee

e-mail: won@kist.re.kr

J.-W. Lee $\cdot$ J. Jang

Department of Physics, Kyunghee University, Seoul 130-701,

Korea

B.-K. Ju

Department of Electrical Engineering, Korea University, Anam-

Dong, Seongbuk-Gu, Seoul 136-701, Korea

e-mail: bkju@korea.ac.kr

Y.-S. Yoon $(\bowtie)$

Department of Advanced Technology Fusion, Konkuk University, Hwayang-dong Gwangjin-gu, Seoul 143-701, Korea

e-mail: ysyoon@konkuk.ac.kr 